\title{
9. OCEANOGRAPHIC OBSERVATIONS IN THE EASTERN INDIAN OCEAN
}

\author{
J. R. Heirtzler, Woods Hole Oceanographic Institution, Woods Hole, Massachusetts
}

Ocean currents, either directly through sediment transport, or indirectly through variations in biological activity in the water, affect the distribution of sediments. Since relatively little is known of ocean currents off western Australia, certain observations relevant to ocean currents were made from Glomar Challenger. These included: (a) surface temperature and salinity measurements from the bow, on a 4-hour basis when the suspended-sediment water sample was taken, and (b) $\mathrm{XBT}^{2}$ readings taken from the stern on an approximate 4-hour basis. Bucket-water temperature was taken from the stern at that time as well, to check the calibration of the XBT. Bow readings were usually taken simultaneously with stern readings.

\section{OCEAN SURFACE CURRENTS}

While drilling, Glomar Challenger should offer an excellent midocean platform for direct observation of ocean currents. However, the instruments to make those observations were not available.

The literature contains little information about currents in this area. Pilot charts for the Indian Ocean (Atlas of Pilot Charts, U.S. Navy, 1966) show that the surface currents off western Australia are controlled by two major current systems:

1) The circum-Antarctic flow south of Australia going from west to east at all times of year, and

2) the currents of the Timor Sea, especially along its northern side, adjacent to Indonesia. These flow into the Indian Ocean, to the west, for all months except March, when flow is to the east.

The currents off western Australia are the result of a shear between these two oppositely directed systems. Since the intensity and geometry, if not direction, of these two systems changes from month to month, the currents in the shear zone change as well.

Off the Australian coast, at least as far west as $110^{\circ} \mathrm{E}$ the predominant direction of the current is to the south. In January and October, however, the flow direction is to the north. Also, in September and November the southern half of the coast experiences currents to the south, and the northern coast currents to the north. Thus, during the time of Leg 27 , the currents were essentially to the south along the coast during the entire time and were to the east between Sites 260 and 262, providing that the Pilot Charts are accurate. Velocities are indicated as being between 13 and $30 \mathrm{~km} /$ day ( 7 and 16 n.m./day). These expected currents do not obviously contradict any experiences on the cruise.

\footnotetext{
'Woods Hole Oceanographic Institution Contribution No. 3199.

${ }^{2} \mathrm{XBT}=$ expendable bathythermograph.
}

\section{SURFACE SALINITY AND TEMPERATURE}

Figure 1 shows observed surface salinity. Values of salinity greater than $35 \%$ are confined to the region south of $26^{\circ} \mathrm{S}$ and east of $111^{\circ} \mathrm{E}$. Values equal to or exceeding $36 \%$ are confined to an even smaller area within about $185 \mathrm{~km}$ (100 n.m.) of Site 259. The Oceanographic Atlas of the Indian Ocean (Wyrtki, 1971) shows $35 \%$ values existing south of about $29^{\circ} \mathrm{S}$ and no $36 \%$ values existing in the area. Thus, the observed values do not agree with those reported by Wyrtki (1971).

Temperatures observed at the surface are shown in Figure 2. The isotherms essentially follow those shown by Wyrtki (1971) with the warm water in excess of $30^{\circ} \mathrm{C}$ in the Timor Sea being somewhat unexpected. A T-S diagram of the surface waters reinforces this tendency of higher temperatures to be associated with lower salinities and lower temperatures to be associated with higher salinities to the south. The T-S diagram does not allow identification of separate water masses in the area.

\section{TEMPERATURE STRUCTURE OF THE UPPER 200 METERS}

Seventy-eight XBT measurements were taken at the positions indicated in Figure 3. The actual XBT records are shown in Figures 4 and 5 and the times and surface readings are given in Table 1 .

The XBT records show a thermocline, usually at about 30 meters $(100 \mathrm{ft})$ in depth. The records also show many small-scale temperature fluctuations with many similar fluctuations persisting onto the next record-a distance of about $90 \mathrm{~km}(50 \mathrm{n} . \mathrm{m}$.). These are probably caused by a localized turbulence phenomenon.

From the individual XBT records it is possible to construct two north-south temperature-depth profiles, one for the northbound leg and one for the southbound legs (Figures 6 and 7). The chief characteristic of the profiles is a rising towards the surface of the isotherms as one proceeds south from about $15^{\circ} \mathrm{S}$. The isotherms are at a more constant level in the area north of about $15^{\circ} \mathrm{S}$, confirming the general observations of Wyrtki (1971). The simplest explanation of these facts is a gentle mixing of water down to 200 meters by flow from the Timor Sea, but less homogenized water off western Australia.

\section{ACKNOWLEDGMENTS}

The U.S. Naval Postgraduate School, Monterey, California, supplied the XBT's, and M. Fields took the XBT readings. $\mathrm{H}$. Hays plotted temperature profiles and assisted with other graphic work. Their efforts are appreciated. 


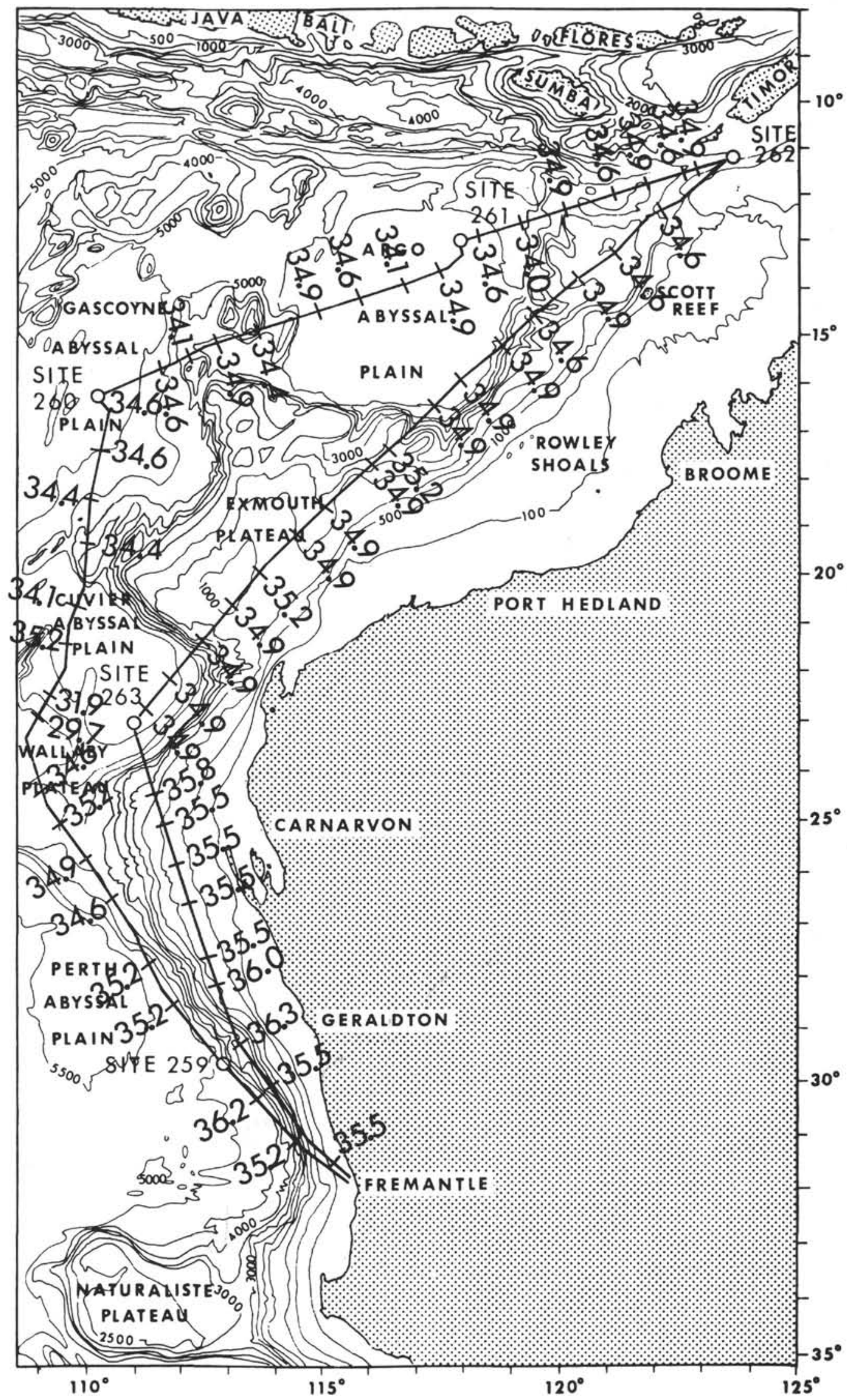

Figure 1. Observed surface salinity, $\%$. 


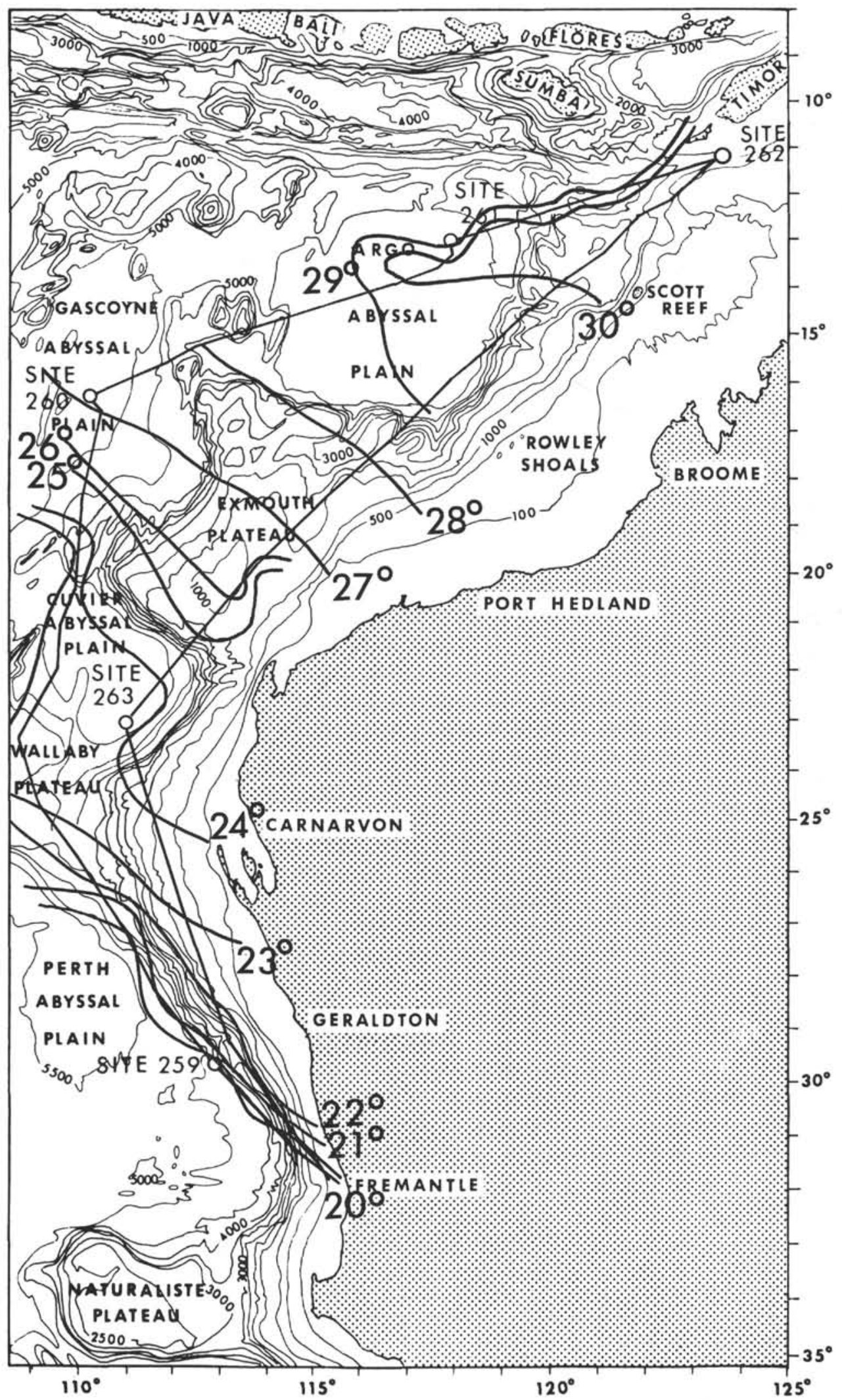

Figure 2. Observed surface temperature, ${ }^{\circ} \mathrm{C}$. 


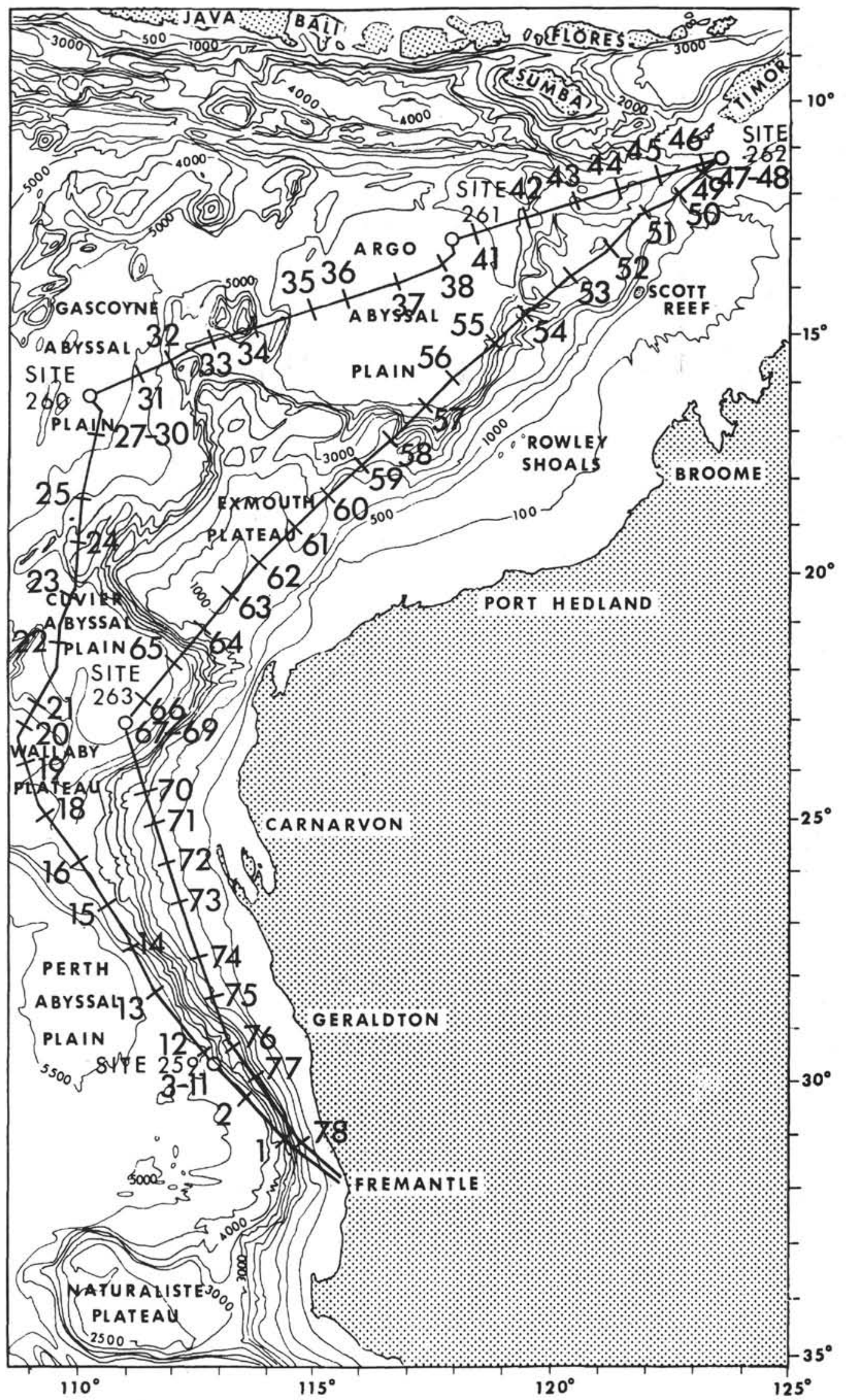

Figure 3. XBT number and locations. 

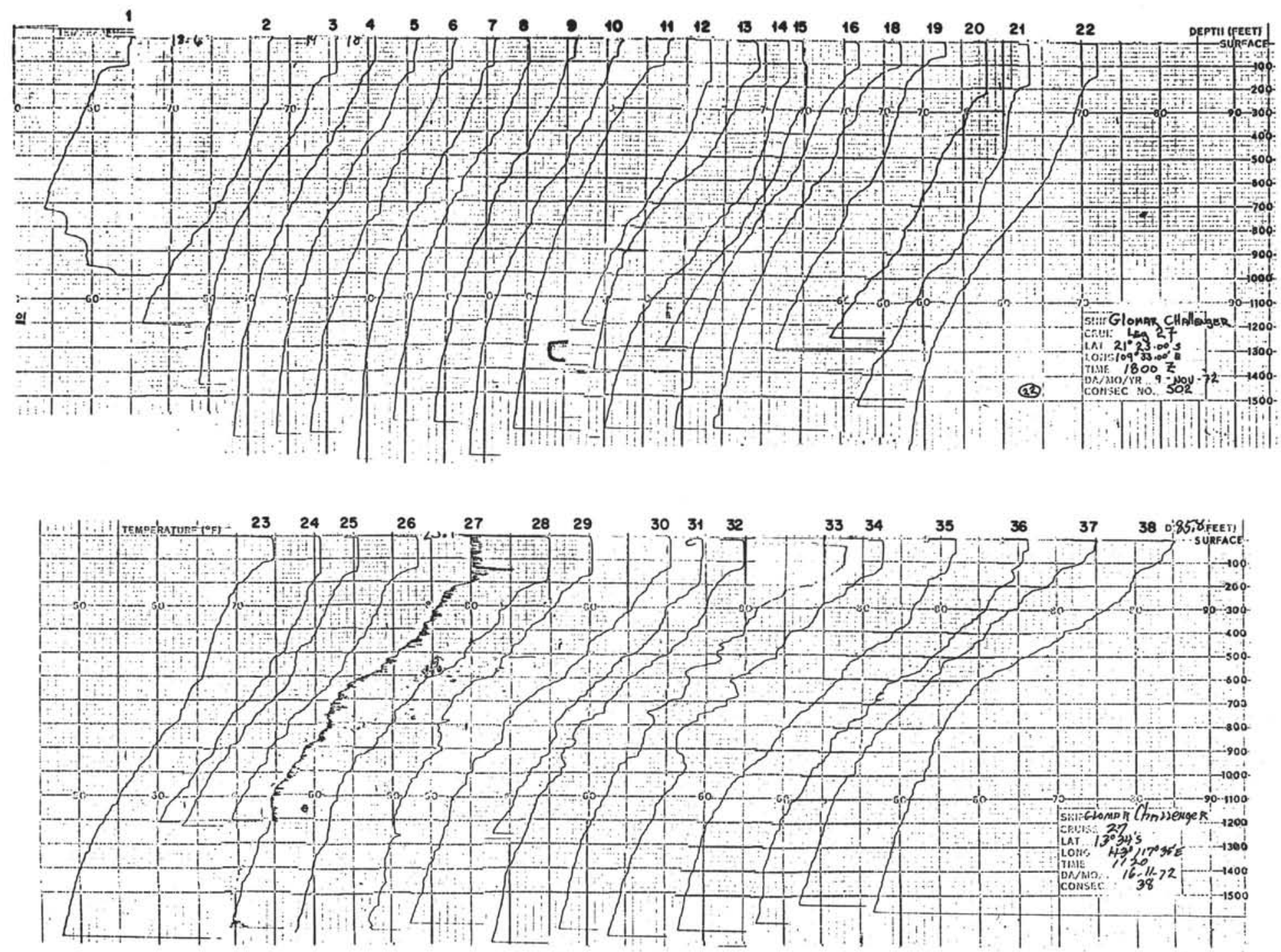

Figure 4. XBT records, depth scale in feet and temperature scale in ${ }^{\circ} \mathrm{F}$. Surface temperature is given in Table 1. 

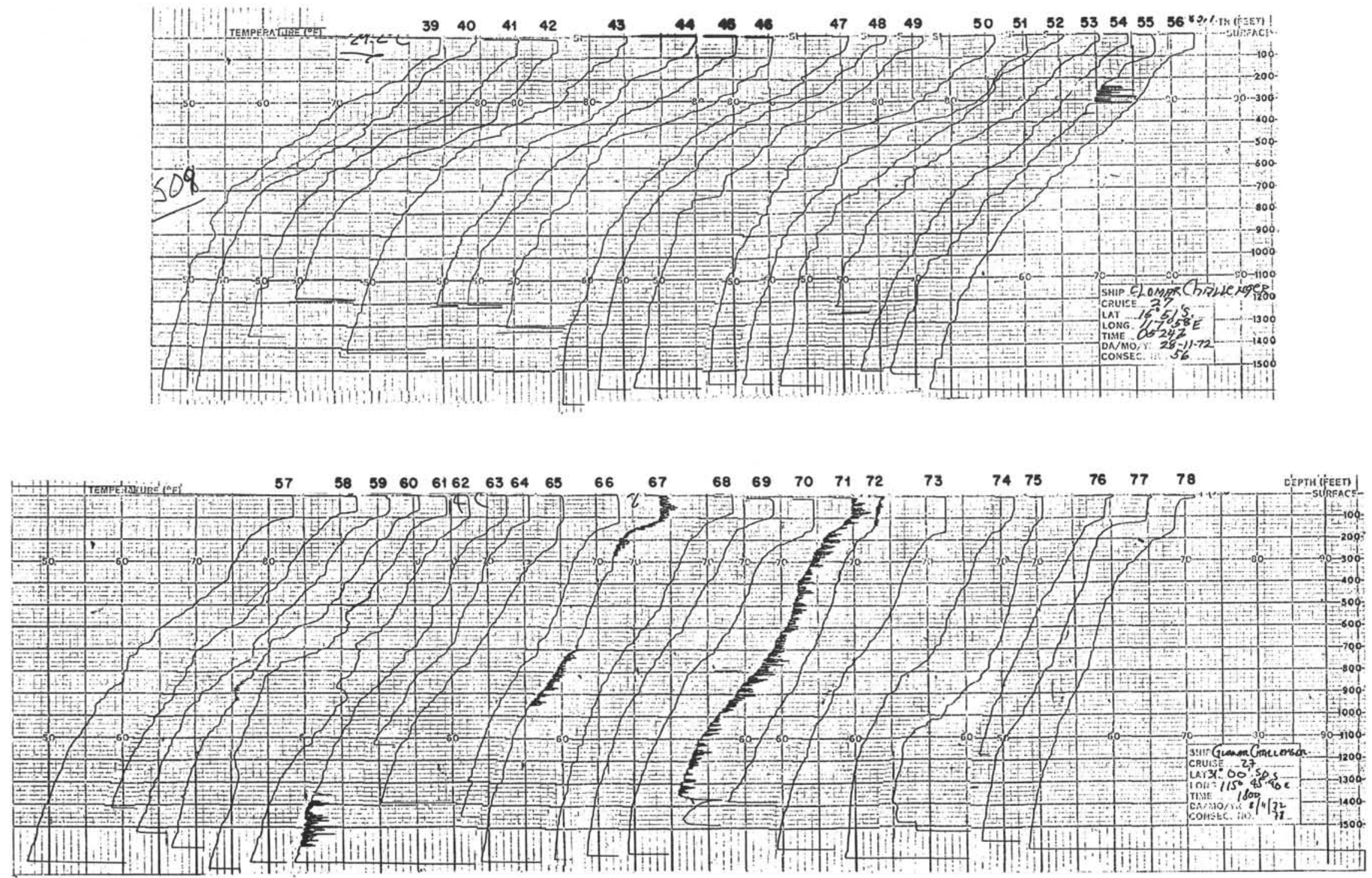

Figure 5. XBT records, depth scale in feet and temperature scale in ${ }^{\circ}$. Surface temperature is given in Table 1. 
TABLE 1

XBT Measurements - Leg 27, Glomar Challenger 2 November-9 December 1972

\begin{tabular}{|c|c|c|c|c|c|c|}
\hline $\begin{array}{l}\text { XBT } \\
\text { No. }\end{array}$ & Date & $\begin{array}{c}\text { Time } \\
\text { (Z) }\end{array}$ & $\begin{array}{c}\text { Stern } \\
\text { Surface } \\
\text { Temp. (F) }\end{array}$ & Latitude & Longitude & $\begin{array}{c}\text { Filter } \\
\text { No. }\end{array}$ \\
\hline 1 & 2 Nov. & 0400 & 65.5 & $31^{\circ} 12^{\prime} \mathrm{S}$ & $114^{\circ} 30^{\prime} \mathrm{E}$ & 2101 \\
\hline 2 & 2 Nov. & 1015 & 68.0 & $30^{\circ} 24^{\prime} \mathrm{S}$ & $113^{\circ} 41^{\prime} \mathrm{E}$ & 2102 \\
\hline 3 & 2 Nov. & 1600 & 66.0 & $29^{\circ} 47^{\prime} \mathrm{S}$ & $112^{\circ} 46^{\prime} \mathrm{E}$ & 2103 \\
\hline 4 & 2 Nov. & 2200 & 66.2 & $29^{\circ} 42^{\prime} \mathrm{S}$ & $112^{\circ} 42^{\prime} \mathrm{E}$ & 2104 \\
\hline 5 & 3 Nov. & 0000 & 66.7 & $29^{\circ} 37^{\prime} \mathrm{S}$ & $112^{\circ} 41^{\prime} \mathrm{E}$ & 2105 \\
\hline 6 & 3 Nov. & 1000 & 65.8 & $29^{\circ} 37^{\prime} \mathrm{S}$ & $112^{\circ} 42^{\prime} \mathrm{E}$ & 2106 \\
\hline 7 & 4 Nov. & 0005 & 66.5 & $29^{\circ} 37^{\prime} \mathrm{S}$ & $112^{\circ} 42^{\prime} \mathrm{E}$ & 2107 \\
\hline 8 & 4 Nov. & 2200 & 64.9 & $29^{\circ} 37^{\prime} \mathrm{S}$ & $112^{\circ} 42^{\prime} \mathrm{E}$ & 2108 \\
\hline 9 & 5 Nov. & 0320 & 66.2 & $29^{\circ} 37^{\prime} \mathrm{S}$ & $112^{\circ} 42^{\prime} \mathrm{E}$ & 2109 \\
\hline 10 & 5 Nov. & 0930 & 67.3 & $29^{\circ} 37^{\prime} \mathrm{S}$ & $112^{\circ} 42^{\prime} \mathrm{E}$ & 2110 \\
\hline 11 & 7 Nov. & 0520 & 68.0 & $29^{\circ} 37^{\prime} \mathrm{S}$ & $112^{\circ} 42^{\prime} \mathrm{E}$ & 2120 \\
\hline 12 & 7 Nov. & 1120 & 68.0 & $29^{\circ} 37^{\prime} \mathrm{S}$ & $112^{\circ} 30^{\prime} \mathrm{E}$ & \\
\hline 13 & 7 Nov. & 1800 & 68.5 & $29^{\circ} 36^{\prime} \mathrm{S}$ & $111^{\circ} 41^{\prime} \mathrm{E}$ & 2124 \\
\hline 14 & 7 Nov. & 2310 & 68.0 & $27^{\circ} 25^{\prime} \mathrm{S}$ & $111^{\circ} 03^{\prime} \mathrm{E}$ & \\
\hline 15 & 8 Nov. & 0510 & 69.8 & $26^{\circ} 38^{\prime} \mathrm{S}$ & $110^{\circ} 33^{\prime} \mathrm{E}$ & \\
\hline 16 & 8 Nov. & 1110 & 71.6 & $25^{\circ} 47^{\prime} \mathrm{S}$ & $110^{\circ} 04^{\prime} \mathrm{E}$ & \\
\hline 17 & & & & & & \\
\hline 18 & 8 Nov. & 1800 & 72.5 & $24^{\circ} 46^{\prime} \mathrm{S}$ & $109^{\circ} 23^{\prime} \mathrm{E}$ & 2118 \\
\hline 19 & 8 Nov. & 2320 & 72.9 & $24^{\circ} 00^{\prime} \mathrm{S}$ & $108^{\circ} 53^{\prime} \mathrm{E}$ & \\
\hline 20 & 9 Nov. & 0530 & 72.9 & $23^{\circ} 09^{\prime} \mathrm{S}$ & $108^{\circ} 32^{\prime} \mathrm{E}$ & 501 \\
\hline 21 & 9 Nov. & 1115 & 73.4 & $22^{\circ} 22^{\prime} \mathrm{S}$ & $109^{\circ} 07^{\prime} \mathrm{E}$ & \\
\hline 22 & 9 Nov. & 1800 & 72.1 & $21^{\circ} 23^{\prime} \mathrm{S}$ & $109^{\circ} 33^{\prime} \mathrm{E}$ & 502 \\
\hline 23 & 9 Nov. & 2320 & 74.8 & $20^{\circ} 30^{\prime} \mathrm{S}$ & $109^{\circ} 45^{\prime} \mathrm{E}$ & \\
\hline 24 & 10 Nov. & 0535 & 76.3 & & & \\
\hline 25 & 10 Nov. & 1120 & 75.6 & $18^{\circ} 25^{\prime} \mathrm{S}$ & $110^{\circ} 10^{\prime} \mathrm{E}$ & \\
\hline 26 & 10 Nov. & 1815 & 78.3 & $17^{\circ} 04^{\prime} \mathrm{S}$ & $110^{\circ} 15^{\prime} \mathrm{E}$ & 504 \\
\hline 27 & 10 Nov. & 2315 & 80.1 & $16^{\circ} 14^{\prime} \mathrm{S}$ & $110^{\circ} 28^{\prime} \mathrm{E}$ & \\
\hline 28 & 11 Nov. & 1125 & 80.2 & $16^{\circ} 09^{\prime} \mathrm{S}$ & $110^{\circ} 18^{\prime} \mathrm{E}$ & 2130 \\
\hline 29 & 13 Nov. & 1210 & 80.3 & $16^{\circ} 09^{\prime} \mathrm{S}$ & $110^{\circ} 18^{\prime} \mathrm{E}$ & \\
\hline 30 & 14 Nov. & 1205 & 80.2 & $16^{\circ} 09^{\prime} \mathrm{S}$ & $110^{\circ} 18^{\prime} \mathrm{E}$ & \\
\hline 31 & 14 Nov. & 1915 & 79.5 & $15^{\circ} 45^{\prime} \mathrm{S}$ & $111^{\circ} 10^{\prime} \mathrm{E}$ & \\
\hline 32 & 14 Nov. & 2325 & 80.1 & $15^{\circ} 31^{\prime} \mathrm{S}$ & $111^{\circ} 39^{\prime} \mathrm{E}$ & 2148 \\
\hline 33 & 15 Nov. & 0517 & 82.9 & $15^{\circ} 10^{\prime} \mathrm{S}$ & $112^{\circ} 42^{\prime} \mathrm{E}$ & 506 \\
\hline 34 & 15 Nov. & 1125 & 82.6 & $14^{\circ} 51^{\prime} \mathrm{S}$ & $113^{\circ} 41^{\prime} \mathrm{E}$ & 2149 \\
\hline 35 & 15 Nov. & 1820 & 81.9 & $14^{\circ} 31^{\prime} \mathrm{S}$ & $114^{\circ} 44^{\prime} \mathrm{E}$ & 507 \\
\hline 36 & 15 Nov. & 2328 & 81.7 & $14^{\circ} 12^{\prime} \mathrm{S}$ & $115^{\circ} 42^{\prime} \mathrm{E}$ & 2150 \\
\hline 37 & 16 Nov. & 0518 & 85.8 & $13^{\circ} 52^{\prime} \mathrm{S}$ & $116^{\circ} 37^{\prime} \mathrm{E}$ & 508 \\
\hline 38 & 16 Nov. & 1120 & 85.8 & $13^{\circ} 34^{\prime} \mathrm{S}$ & $117^{\circ} 35^{\prime} \mathrm{E}$ & 2151 \\
\hline 39 & 16 Nov. & 1800 & 84.6 & $12^{\circ} 56^{\prime} \mathrm{S}$ & $117^{\circ} 51^{\prime} \mathrm{E}$ & 509 \\
\hline 40 & 22 Nov. & 0001 & 84.4 & $12^{\circ} 56^{\prime} \mathrm{S}$ & $117^{\circ} 51^{\prime} \mathrm{E}$ & 2152 \\
\hline 41 & 22 Nov. & 0525 & 85.8 & $12^{\circ} 46^{\prime} \mathrm{S}$ & $118^{\circ} 34^{\prime} \mathrm{E}$ & 510 \\
\hline 42 & 22 Nov. & 1110 & 86.0 & $12^{\circ} 28^{\prime} \mathrm{S}$ & $119^{\circ} 26^{\prime} \mathrm{E}$ & 2173 \\
\hline 43 & 22 Nov. & 1800 & 85.5 & $12^{\circ} 03^{\prime} \mathrm{S}$ & $120^{\circ} 30^{\prime} \mathrm{E}$ & 511 \\
\hline 44 & 22 Nov. & 2320 & 84.6 & $11^{\circ} 49^{\prime} \mathrm{S}$ & $121^{\circ} 19^{\prime} \mathrm{E}$ & \\
\hline 45 & 23 Nov. & 0520 & 85.8 & $11^{\circ} 34^{\prime} \mathrm{S}$ & $122^{\circ} 19^{\prime} \mathrm{E}$ & 512 \\
\hline 46 & 23 Nov. & 1115 & 86.2 & $11^{\circ} 15^{\prime} \mathrm{S}$ & $123^{\circ} 13^{\prime} \mathrm{E}$ & 2175 \\
\hline 47 & 23 Nov. & 1800 & 86.2 & $10^{\circ} 51^{\prime} \mathrm{S}$ & $123^{\circ} 53^{\prime} \mathrm{E}$ & 513 \\
\hline 48 & 25 Nov. & 2330 & 86.4 & $10^{\circ} 51^{\prime} \mathrm{S}$ & $123^{\circ} 53^{\prime} \mathrm{E}$ & 2184 \\
\hline 49 & 26 Nov. & 1115 & 86.7 & $11^{\circ} 16^{\prime} \mathrm{S}$ & $123^{\circ} 25^{\prime} \mathrm{E}$ & 2185 \\
\hline 50 & 26 Nov. & 1800 & 86.7 & $12^{\circ} 06^{\prime} \mathrm{S}$ & $122^{\circ} 29^{\prime} \mathrm{E}$ & 515 \\
\hline 51 & 26 Nov. & 2325 & 85.4 & $12^{\circ} 41^{\prime} \mathrm{S}$ & $121^{\circ} 45^{\prime} \mathrm{E}$ & \\
\hline 52 & 27 Nov. & 0522 & 85.6 & $13^{\circ} 20^{\prime} \mathrm{S}$ & $120^{\circ} 59^{\prime} \mathrm{E}$ & 516 \\
\hline 53 & 27 Nov. & 1120 & 85.5 & $13^{\circ} 52^{\prime} \mathrm{S}$ & $120^{\circ} 14^{\prime} \mathrm{E}$ & 2187 \\
\hline 54 & 27 Nov. & 1800 & 84.2 & $14^{\circ} 32^{\prime} \mathrm{S}$ & $119^{\circ} 20^{\prime} \mathrm{E}$ & 517 \\
\hline 55 & 27 Nov. & 2320 & 83.3 & $15^{\circ} 09^{\prime} \mathrm{S}$ & $118^{\circ} 45^{\prime} \mathrm{E}$ & 2188 \\
\hline 56 & 28 Nov. & 0524 & 83.7 & $15^{\circ} \mathrm{S} 1^{\prime} \mathrm{S}$ & $117^{\circ} 58^{\prime} \mathrm{E}$ & 518 \\
\hline 57 & 28 Nov. & 1115 & 83.3 & $16^{\circ} 25^{\prime} \mathrm{S}$ & $117^{\circ} 19^{\prime} \mathrm{E}$ & 2189 \\
\hline 58 & 28 Nov. & 1800 & 82.1 & $17^{\circ} 26^{\prime} \mathrm{S}$ & $116^{\circ} 24^{\prime} \mathrm{E}$ & 519 \\
\hline 59 & 28 Nov. & 2320 & 81.3 & $17^{\circ} 45^{\prime} \mathrm{S}$ & $115^{\circ} 57^{\prime} \mathrm{E}$ & 2190 \\
\hline 60 & 29 Nov. & 0533 & 81.0 & $18^{\circ} 21^{\prime} \mathrm{S}$ & $115^{\circ} 13^{\prime} \mathrm{E}$ & 520 \\
\hline
\end{tabular}


TABLE 1 - Continued

\begin{tabular}{ccccccr}
\hline $\begin{array}{c}\text { XBT } \\
\text { No. }\end{array}$ & Date & $\begin{array}{c}\text { Time } \\
(\mathrm{Z})\end{array}$ & $\begin{array}{c}\text { Stern } \\
\text { Surface } \\
\text { Temp. }\left({ }^{\circ} \mathrm{F}\right)\end{array}$ & Latitude & Longitude & $\begin{array}{r}\text { Filter } \\
\text { No. }\end{array}$ \\
\hline 61 & 29 Nov. & 1115 & 79.7 & $18^{\circ} 55^{\prime} \mathrm{S}$ & $114^{\circ} 35^{\prime} \mathrm{E}$ & 2191 \\
62 & 29 Nov. & 1800 & 77.7 & $19^{\circ} 48^{\prime} \mathrm{S}$ & $113^{\circ} 47^{\prime} \mathrm{E}$ & 521 \\
63 & 29 Nov. & 2318 & 77.9 & $20^{\circ} 26^{\prime} \mathrm{S}$ & $113^{\circ} 14^{\prime} \mathrm{E}$ & \\
64 & 30 Nov. & 0519 & 76.7 & $21^{\circ} 10^{\prime} \mathrm{S}$ & $112^{\circ} 37^{\prime} \mathrm{E}$ & 522 \\
65 & 30 Nov. & 1120 & 75.6 & $21^{\circ} 54^{\prime} \mathrm{S}$ & $111^{\circ} 56^{\prime} \mathrm{E}$ & \\
66 & 30 Nov. & 1800 & 73.4 & $22^{\circ} 42^{\prime} \mathrm{S}$ & $111^{\circ} 17^{\prime} \mathrm{E}$ & 523 \\
67 & 30 Nov. & 2305 & 73.4 & $23^{\circ} 12^{\prime} \mathrm{S}$ & $110^{\circ} 51^{\prime} \mathrm{E}$ & \\
68 & 3 Dec. & 2330 & 73.4 & $23^{\circ} 19^{\prime} \mathrm{S}$ & $110^{\circ} 57^{\prime} \mathrm{E}$ & 2205 \\
69 & 6 Dec. & 0620 & 74.5 & $23^{\circ} 19^{\prime} \mathrm{S}$ & $110^{\circ} 57^{\prime} \mathrm{E}$ & \\
70 & 6 Dec. & 1800 & 75.2 & $24^{\circ} 25^{\prime} \mathrm{S}$ & $111^{\circ} 24^{\prime} \mathrm{E}$ & 524 \\
71 & 6 Dec. & 2320 & 74.8 & $25^{\circ} 04^{\prime} \mathrm{S}$ & $111^{\circ} 37^{\prime} \mathrm{E}$ & \\
72 & 7 Dec. & 0520 & 73.8 & $25^{\circ} 49^{\prime} \mathrm{S}$ & $111^{\circ} 56^{\prime} \mathrm{E}$ & 525 \\
73 & 7 Dec. & 1120 & 73.4 & $26^{\circ} 37^{\prime} \mathrm{S}$ & $112^{\circ} 15^{\prime} \mathrm{E}$ & \\
74 & 7 Dec. & 1800 & 72.0 & $27^{\circ} 45^{\prime} \mathrm{S}$ & $112^{\circ} 43^{\prime} \mathrm{E}$ & 526 \\
75 & 7 Dec. & 2330 & 71.2 & $28^{\circ} 26^{\prime} \mathrm{S}$ & $112^{\circ} 56^{\prime} \mathrm{E}$ & \\
76 & 8 Dec. & 0540 & 70.3 & $29^{\circ} 23^{\prime} \mathrm{S}$ & $113^{\circ} 27^{\prime} \mathrm{E}$ & \\
77 & 8 Dec. & 1120 & 71.6 & $30^{\circ} 05^{\prime} \mathrm{S}$ & $114^{\circ} 02^{\prime} \mathrm{E}$ & 2218 \\
78 & 8 Dec. & 1800 & 71.2 & $31^{\circ} 00^{\prime} \mathrm{S}$ & $115^{\circ} 45^{\prime} \mathrm{E}$ & 528 \\
\hline
\end{tabular}

\section{REFERENCES}

U.S. Naval Oceanographic office, 1966. Atlas of pilot charts of South Pacific and Indian oceans, H. O. Publ. No. 109 (3rd Ed.): Washington (U.S. Government Printing Office).

Wyrtki, K., 1971. Oceanographic Atlas of the International Indian Ocean Expedition: Washington (U.S. Government Printing Office). 


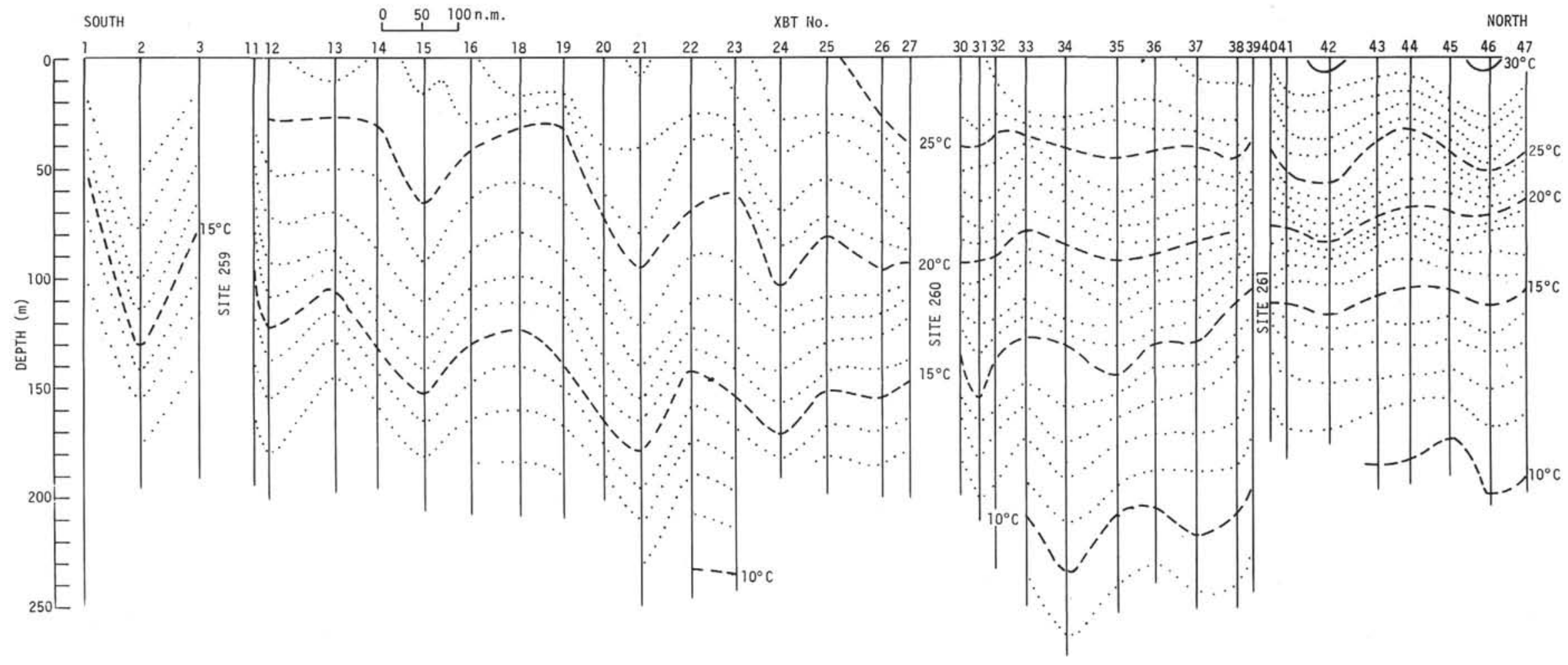

Figure 6. Temperature depth profile for the northerly track, depth in meters and temperature in ${ }^{\circ} \mathrm{C}$. 


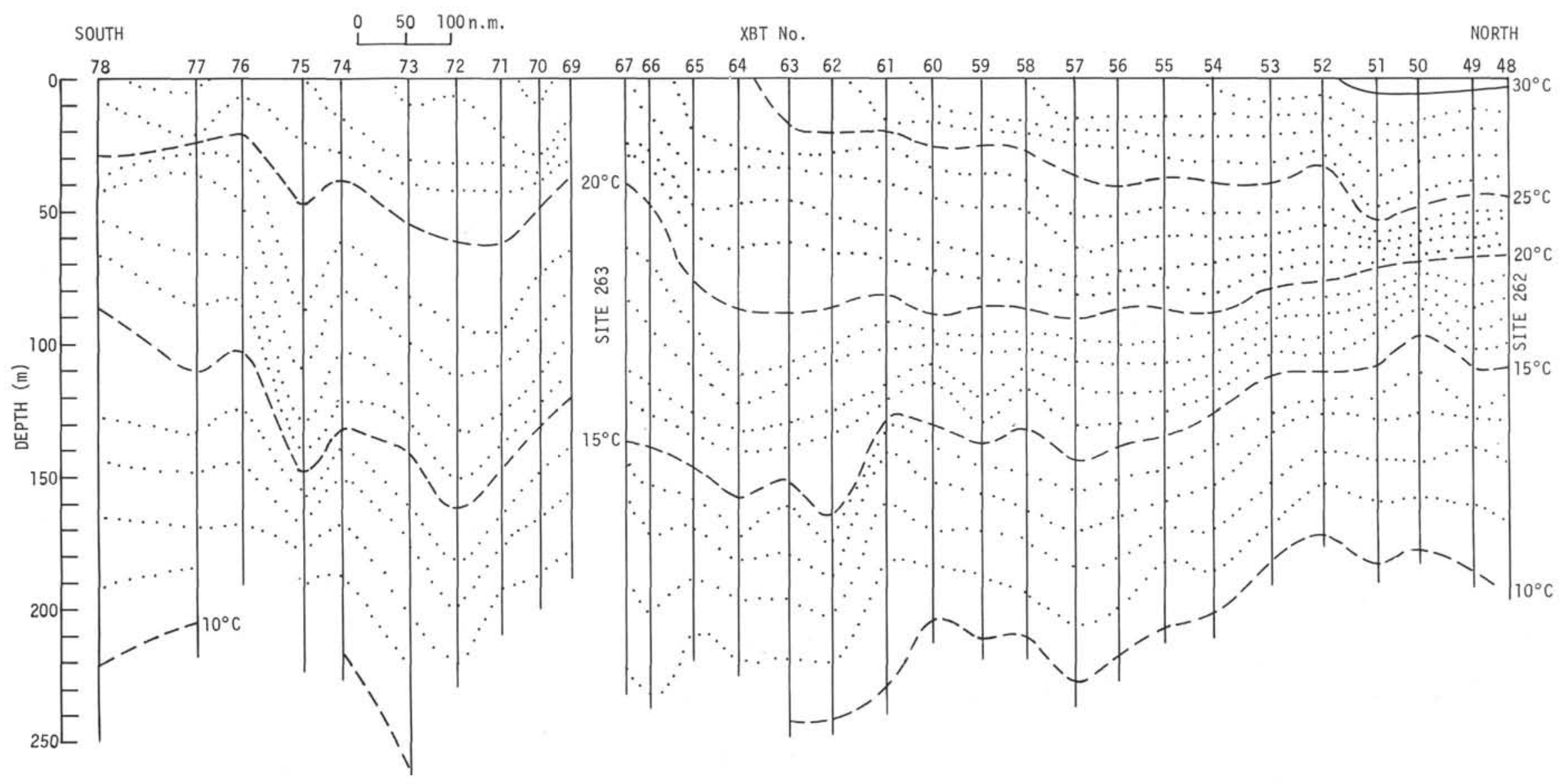

Figure 7. Temperature depth profile for the southerly track, depth in meters and temperature in ${ }^{\circ} \mathrm{C}$. 